\title{
Colitis cystica profunda complicated by complete colorectal obstruction
}

\author{
HUGH JAMES FREEMAN MD
}

HJ FreEmAN. Colitis cystica profunda complicated by complete colorectal obstruction. Can J Gastroenterol 1994;8(5):326-330. An unusual presentation of colitis cystica profunda was seen in a 39 -year-old male with a complete colonic obstruction due to an intussception with a rectosigmoid inflammatory mass. The patient had apparent antecedent distal ulcerative colitis and management included subtotal colectomy. Removal of the rectosigmoid mass produced a satisfactory clinical result with no colonoscopic or histological evidence of recurrent disease in the subsequent decade. Although rare, this entity should be considered during the evaluation of any inflammatory process involving the distal colon, especially if an inflammatory polypoid colonic mass is present or a mucinous adenocarcinoma is suspected.

Key Words: Colitis cystica profunda, Colonic obstruction, Distal colitis, Inflammatory bowel disease, Ulcerative colitis

\section{Colite kystique profonde compliquée par une obstruction colorectale totale}

RÉSUMÉ : Un tableau inhabituel de colite kystique profonde a été observé chez un homme de 39 ans atteint d'une obstruction colique totale attribuable à une intussusception avec masse inflammatoire rectosigmoïdienne. Le patient avait manifestement des signes de colite ulcéreuse distale et avait été traité, entre autre, par colectomie subtotale. La résection de la masse rectosigmoïdienne a donné des résultats cliniques satisfaisants sans signes coloscopiques ou histologiques de maladie récidivante au cours de la décennie suivante. Bien que rare, cette entité doit être envisagée durant l'évaluation de tout processus inflammatoire qui met en jeu le côlon distal, particulièrement en présence d'une masse colique polypoïde inflammatoire ou si l'on soupçonne un adénocarcinome mucinö̈de.

Department of Medicine (Gastroenterology), University Hospital and University of British Columbia, Vancouver, British Columbia

Correspondence and reprints: Dr Hugh Freeman, Head, Gastroenterology, ACU F-137, University Hospital (UBC site), 2211 Wesbrook Mall, Vancouver, British Columbia

V6T IW5. Telephone (604) 822-7216

Received for publication November 25, 1992. Accepted March 3, 1993 $\checkmark$ OLITIS CYSTICA PROFUNDA IS AN unusual inflammatory disorder of the colon consisting of benign epithelial lined mucus cysts in the mucosa and submucosa, leading to polypoid colonic inflammatory masses (1-5). It differs from a rarer disorder, colitis cystica superficialis, that is entirely mucosal and has been associated with pellagra (3). Fewer than 100 cases have been described in the literature and a number of different types have resulted in a classification (5) that includes a localized form, almost always in the rectum, a segmental form that may involve one or more bowel segments and a diffuse form with lesions scattered throughout the colon. The polypoid lesions vary substantially in size from millimetres to centimetres and appear as broad-based polyps, nodules or papillary masses. Colitis cystica profunda may be confused with a well-differentiated adenocarcinoma invading the muscularis mucosa but the distinctive characteristic of this disorder is the entirely benign cytological appearance of the colonocytes (6). The pathogenesis of the lesion is not clear and most common clinical features include diarrhea, abdominal cramps and pain as well as passage of blood and mucus in the 

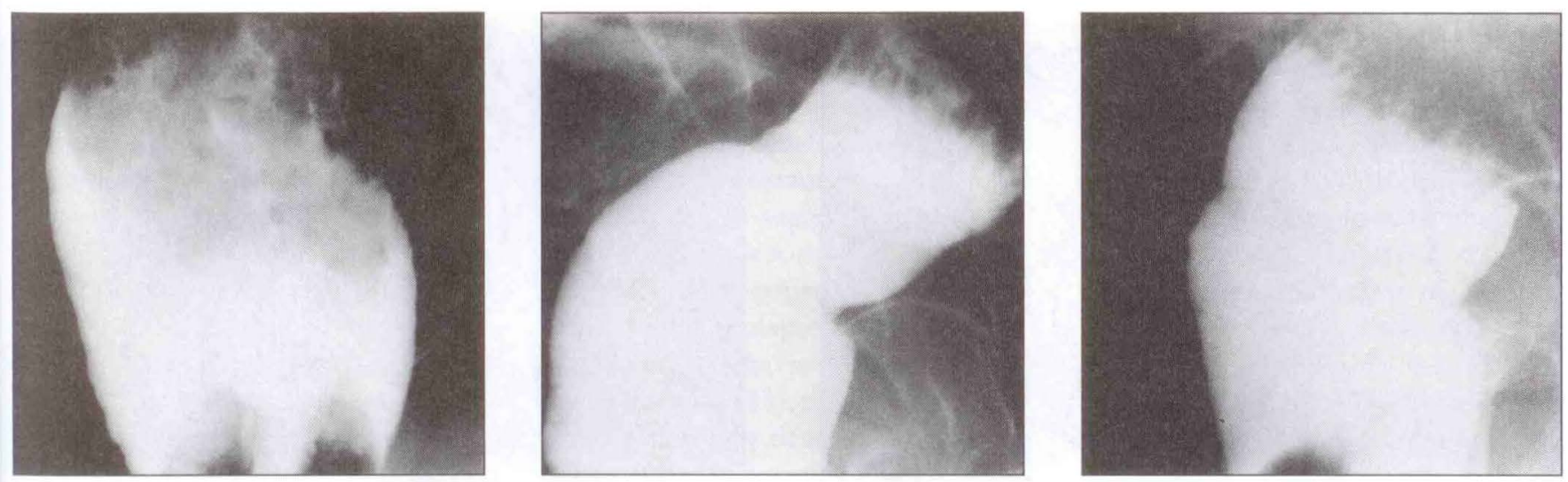

Figure 1) Barium enema in different projections showing a rectal mass with complete colonic obstruction

stools. Anemia and hypoalbuminemia may develop. Endoscopic or radiological studies may reveal polypoid or thickened mucosal folds. Localized excision or limited resection has been the recommended treatment.

The patient described in the present report is of special interest because of the apparent preceding features of distal and localized ulcerative colitis resulting in the development of a polypoid inflammatory mass, subsequent intussception and complete colonic obstruction. Diagnostic confusion could arise in this setting with any mucosal inflammatory process for the clinical gastroenterologist since endoscopic pinch biopsies may not be sufficient to obtain tissue from the deeper submucosal region. In addition, concern has previously been raised regarding the possible difficulties in differentiating this condition from a mucin-secreting carcinoma.

\section{CASE PRESENTATION}

A 39-year-old male was evaluated in December 1982 because of an eightmonth history of intermittent diarrhea and rectal bleeding associated with a $5 \mathrm{~kg}$ weight loss. Examination was normal. Laboratory studies revealed: hemoglobin, $148 \mathrm{~g} / \mathrm{L}$; white blood cell count, $5.9 \times 10^{9} / \mathrm{L}$; platelets, $237 \times 10^{9} / \mathrm{L}$; erythrocyte sedimentation rate, 35 $\mathrm{mm} / \mathrm{h}$ (normal 0 to 15 ); and normal serum and liver chemistry tests, including serum albumin, $42 \mathrm{~g} / \mathrm{L}$. Fecal cultures for Salmonella, Shigella, Campylobacter and Yersinia species as well as studies for ova and parasites were nega- tive. Colonoscopy revealed diffusely friable mucosa in the distal $35 \mathrm{~cm}$ of colorectum with loss of the normal vascular pattern and contact bleeding. Multiple endoscopic biopsies revealed diffuse mucosal changes in this distal colonic segment with an acute and chronic inflammatory cellular infiltrate as well as crypt abscesses; no granulomas were present. The changes were considered to be consistent with distal ulcerative proctocolitis. Treatment with sulphasalazine $2 \mathrm{~g}$ daily and prednisone $40 \mathrm{mg}$ daily lead to complete resolution of bloody diarrhea and improved endoscopic appearances although additional colonoscopic biopsies showed persistent inflammatory changes. After tapering prednisone over the next eight weeks, the corticosteroid was discontinued.

In August 1983, the patient was reevaluated because of recurrent bloody diarrhea up to six times per day with urgency but no tenesmus. Examination was normal. Laboratory studies revealed a hemoglobin of $131 \mathrm{~g} / \mathrm{L}$ and an erythrocyte sedimentation rate of 61 $\mathrm{mm} / \mathrm{h}$ but other hematology and chemistry tests were normal. Colonoscopy and biopsies revealed diffusely abnormal inflamed mucosa in the distal 35 $\mathrm{cm}$; proximal to this, the mucosa was completely normal. The mucosa in this distal segment was swollen, friable and thickened with apparent inflammatory pseudopolyps. Prednisone $40 \mathrm{mg}$ daily was administered and tapered to $20 \mathrm{mg}$ daily but bloody diarrhea continued with a further fall in the hemoglobin to $112 \mathrm{~g} / \mathrm{L}$.
Because of continued bloody diarrhea, the patient was hospitalized in November 1983. A marked hypochromic microcytic anemia (hemoglobin, $74 \mathrm{~g} / \mathrm{L}$ ) was present with iron deficiency ( $6 \%$ saturation) and hypoalbuminemia (31 g/L). Upper gastrointestinal endoscopy and duodenal biopsies were normal but colonoscopic findings, including biopsies, remained unchanged. The patient was transfused, prednisone $20 \mathrm{mg}$ daily was continued along with sulphasalazine $2 \mathrm{~g}$ daily, and oral iron replacement (ferrous sulphate, $300 \mathrm{mg}$ tid) was provided. Over the next two months, his symptoms gradually resolved and blood tests became normal. The prednisone was tapered and discontinued; he remained completely well on maintenance sulphasalazine $2 \mathrm{~g}$ daily. In March 1984, recurrent diarrhea prompted the patient to request a second opinion from another gastroenterologist. A barium enema was interpreted to show mucosal ulceration in the distal colon and rectum; the proximal colon was normal. Colonoscopy and biopsies confirmed the previous observations. Symptoms resolved and no changes in treatment were required.

In September 1984, the patient presented to the surgical service of another teaching hospital with a mass that the patient thought was a prolapsing hemorrhoid. His bowel habit had changed dramatically over the preceding week; increased mucus was reported with bleeding from the rectum but little feces. Examination revealed a large rectal 


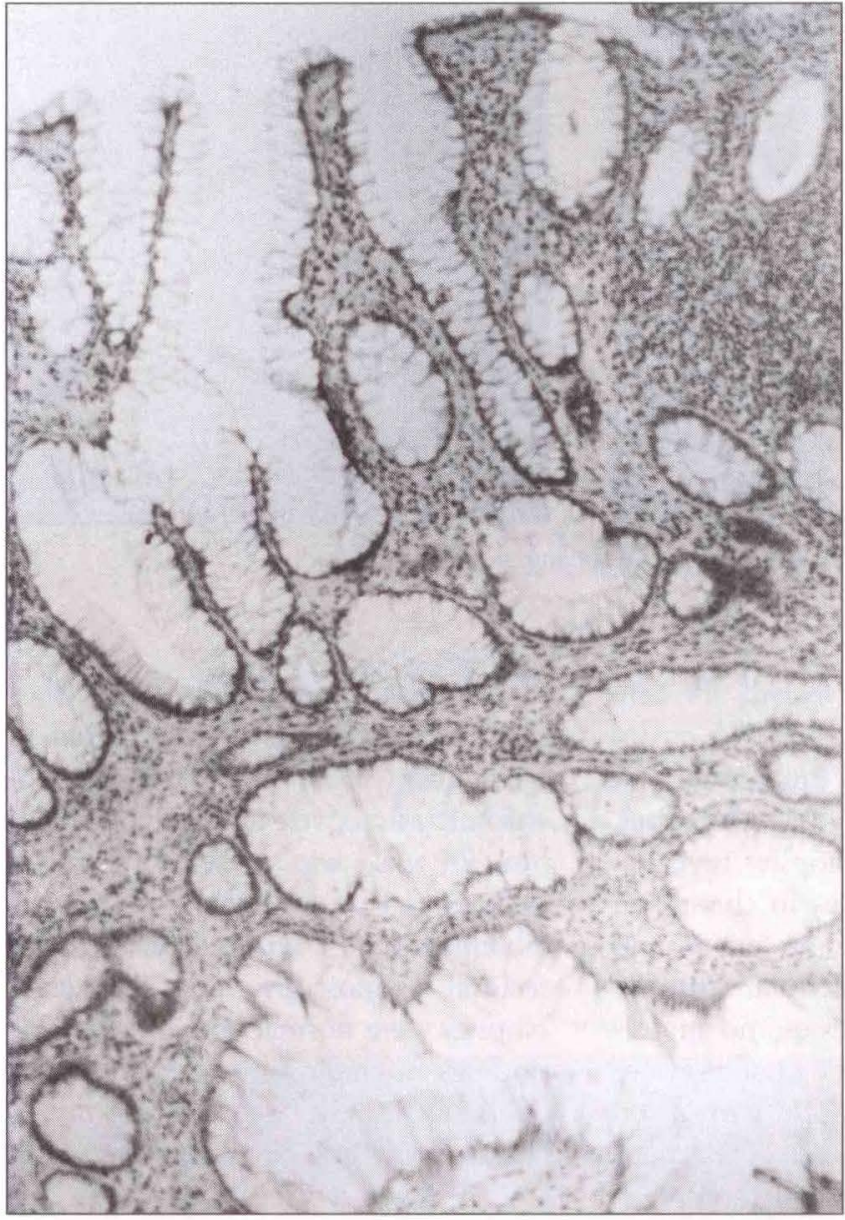

Figure 2) Photomicrograph of the colonic surgical resection specimen from September 1984. Colonic mucosa is seen with an inflammatory infiltrate in the lamina propria and markedly branched colonic crypts. Deep to the mucosa are cystic structures lined by benign colonic epithelium

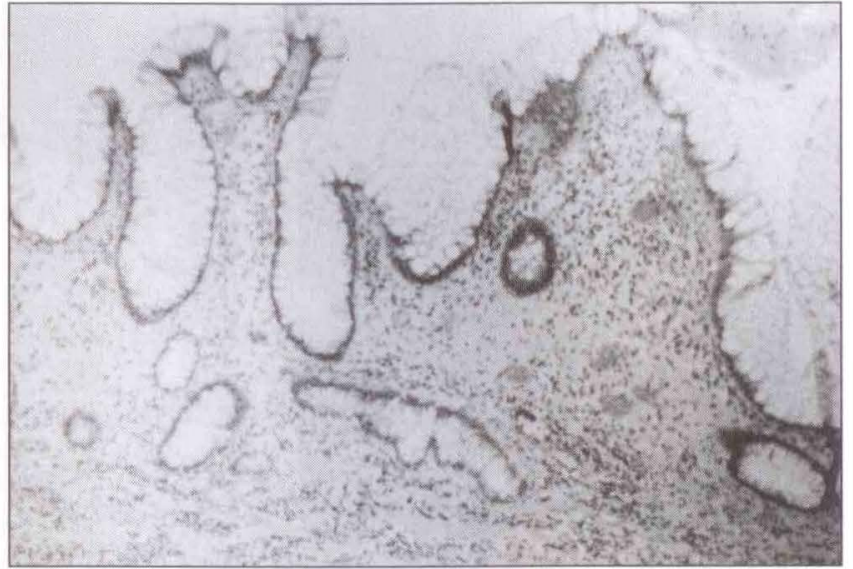

Figure 3) Photomicrograph of the colonic surgical resection specimen from September 1984. Colonic mucosa is seen with branching and marked distortion of colonic crypts, one lying horizontal to the usual crypt axis

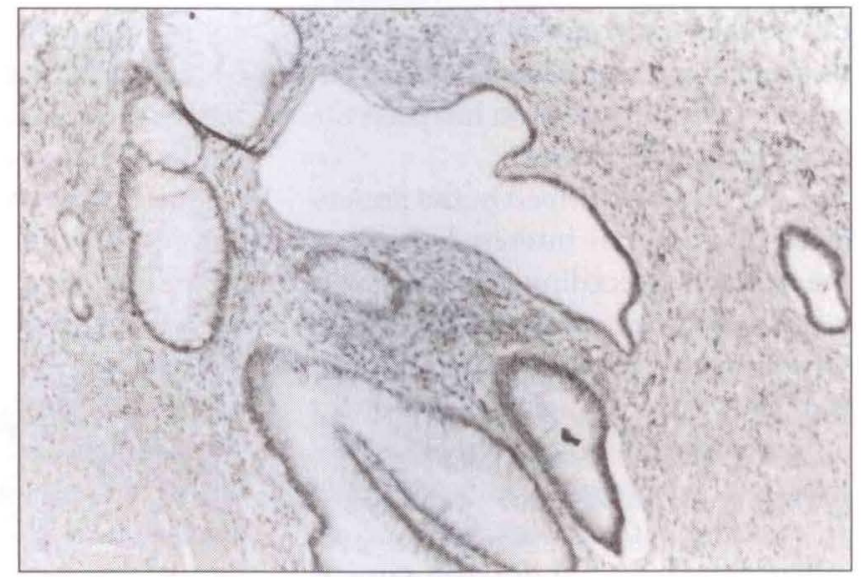

Figure 4) Photomicrograph of the colonic surgical resection specimen from September 1984. Submucosal cysts are seen with benign columnar and cuboidal colonocyte epithelial cells lining mucus-filled cysts tumour protruding through the anus; sigmoidoscopy suggested an obstructing and prolapsing sigmoid tumour with a sigmoidorectal intussusception. Biopsies (frozen and routinely fixed) revealed inflamed polypoid mucosa but no neoplasm. A single contrast barium enema (Figure 1) was attempted but a complete study was not possible because of colonic obstruction associated with a rectosigmoid intussception. It was suggested that this was due to ' $a$ neoplasm or polypoid mucosal surface associated with ulcerative colitis'. Contrast would not enter the proximal colon. Laparotomy revealed a large sigmoid tumour intusscepting into the rectum; the proximal colon was completely normal. A limited (Hartman's type) rectosigmoid resection was done with a left-sided colostomy and crea- tion of a rectal stump. The proximal portion of the resected specimen was entirely normal, but in the distal portion, the mucosa was thickened and firm with a papillary appearance. No neoplastic changes were present. Changes were initially interpreted as consistent with ulcerative colitis and inflammatory pseudopolyposis. After surgery, the patient received only sulphasalazine $2 \mathrm{~g}$ daily. He was reviewed in April 1985 and was completely well. Colonoscopy was done through the stoma along with endoscopic evaluation of the rectal stump. The rectal stump mucosa and colonic mucosa for 4 to $5 \mathrm{~cm}$ proximal to the colostomy were swollen and erythematous with minimal friability; there was no pseudopolypoid change and the proximal colonic mucosa from cecum to within 4 to $5 \mathrm{~cm}$ of the colostomy stoma was completely normal. Biopsies revealed chronic inflammatory changes with branched glands in the rectal pouch and in the most distal colonic mucosal biopsies proximal to the stoma. Biopsies from multiple sites elsewhere in the cecum, ascending colon, transverse colon and descending colon were completely normal. In May 1985, the distal rectum was reconnected to the descending colon and the colostomy was removed. Colonoscopy and multiple colonic mucosal biopsies were done in September 1985; these were normal except for some minimal anastomotic inflammatory changes. The patient remained completely asymptomatic. Repeated colonoscopic evaluations with multiple colonic mucosal biopsies done in October 1986, December 1987 and February 
1991 were normal and sulphasalazine was discontinued. In February 1992, the patient was last reviewed. He had no symptoms off all of his medications; colonoscopy and multiple colonic biopsies were normal.

In March 1991, the patient's prior surgical colectomy sections from September 1984 and May 1985 were reviewed. The papillary tumour-like mass consisted of variable sized mucus-containing cysts primarily in the submucosa; these cysts were mucus-filled and lined with cytologically normal colonic epithelium. A dense inflammatory cell infiltrate was present in the lamina propria consisting of lymphocytes, plasma cells and neutrophils. Crypt abscesses and other nonspecific changes consistent with an inflammatory colonic process were present. However, there was no evidence of neoplastic change. These histopathological features (Figures 2-4) are characteristic of colitis cystica profunda (7). Subsequently, all serially sectioned biopsies from normal and abnormal colonic epithelium obtained during sigmoidoscopic or colonoscopic studies were reviewed. There were 66 endoscopic pinch biopsies done. Twenty-six were obtained from the abnormally inflamed colonic segment in the distal $35 \mathrm{~cm}$ of colorectum during endoscopic procedures done prior to the surgical resection. Of these, 16 contained submucosa and 10 contained only mucosa with or without the muscularis mucosae; no biopsy had histological features that would have resulted in a change in the initial pathological diagnosis to colitis cystica profunda.

\section{DISCUSSION}

Proctocolitis cystica profunda is a distinctly unusual and uncommon inflammatory process that occurs in the colon and rectum. Although it has been reported to complicate pre-existent and more common inflammatory processes, such as ulcerative colitis, Crohn's colitis or radiation-induced colonic mucosal injury, the documentation is limited (8-11). In addition to the inflammatory changes in the mucosa in colitis cystica profunda, benign epithelial-lined mucus cysts usually de- velop in the submucosa; these produce polypoid changes or colonic masses. Most frequently, a solitary rectal or rectosigmoid mass develops that may have a papillary appearance, as in the presented patient, suggestive of either a neoplastic process or colonic pseudopolyps. In some patients with distal disease, rectal prolapse may occur (12) and, very rarely, as recorded in one prior instance (13), complete intestinal obstruction may develop necessitating surgical decompression. In the current report, the presentation was dramatic with sigmoidorectal intussception of an apparent colonic papillary mass lesion; the gross appearance raised the possibility of a neoplastic complication of colitis, a common difficulty noted in the clinical and pathological literature $(6,13)$. In some situations, resective surgery may not be easily avoided, especially if exclusion of a malignant neoplasm becomes an issue. Difficulties arise because of problems in recognition of submucosal mucus-filled cysts below the muscularis mucosae in superficial endoscopic pinch biopsies. Regenerative epithelial changes in mucus-filled cysts, especially with an active inflammatory process, may make distinction of 'pseudoinvasion' from a more sinister process, such as a mucinous colonic adenocarcinoma invading the submucosa, exceedingly difficult. The clinical course of the patient described in this report was especially unusual because of his rather dramatic presentation with an intestinal obstruction. Urgent surgical treatment, including decompression, was necessitated. Efforts in this setting to exclude a malignant process, including frozen sections done on the rectal mass, would not likely have changed the surgical approach, even if an earlier diagnosis of colitis cystica profunda had been established.

The inflammatory process in proctocolitis cystica profunda has been classified into different forms: localized, diffuse or segmental. Pathologically, the most common is the localized variety (13), apparently on the anterior rectal wall within $12 \mathrm{~cm}$ of the anal verge; interestingly, a single report of disease localized to the descending co- lon alone has also appeared (14). Several cases of so-called segmental disease have been described $(4,5,14)$. This type consists of multiple polypoid masses present in a multicentric distribution in one or more segments with normal intervening colonic mucosa; this form may occur in the setting of ulcerative colitis (15). Finally, cases of the diffuse form have also been reported $(8,9,16)$. Many of these have also been reported in association with apparent ulcerative colitis. In all types, macroscopic lesions are either broad-based polyps, nodules or papillary masses with typical microscopic descriptions consisting of mucus-filled cysts below the muscularis mucosae; often surgical specimens are required for accurate recognition. Most important, colonocytes lining the mucus-filled cysts are benign with no evidence of cytological atypia but, occasionally, there may be confusion with a well-differentiated mucussecreting adenocarcinoma invading the muscularis mucosae, a condition well known to complicate the clinical course of long-standing and extensive ulcerative colitis.

The pathogenesis of this unusual form of colitis still requires elucidation. The lesion may represent an unusual mucosal reparative response with downwards extension of regenerating colonic epithelium following mucosal ulceration and production of submucosal cysts (17). This might occur in the setting of ulcerative colitis or any other inflammatory colonic disorder. Alternatively, submucosal epithelial herniation associated with weakness in the muscularis mucosae due to the inflammatory process has been suggested (4). In the majority of recorded cases, a pre-existent colonic inflammatory process has been present, such as ulcerative colitis, and, in some instances, especially if localized to rectum, a close etiological relationship with the solitary rectal ulcer syndrome has also been hypothesized $(12,15,18)$. The key histopathological feature in colitis cystica profunda is a mucosal inflammatory process with downwards extension and the detection of mucus-filled cysts in the submucosa. Other causes of colonic submucosal cysts (in addition to colitis 
cystica profunda) have been previously enumerated (12). These include: infectious colitis, polyps (adenomatous, juvenile, hamartomatous), ureterocolic anastomoses and other surgical procedures, including colostomy. In addition, other extracolonic sites may be involved in the gastrointestinal and biliary tract with a similar process, especially in the setting of prior gastrointestinal surgery with anastomoses. Mucosa

\section{REFERENCES}

1. Tedesco FJ, Sumner HW, Kassens YM. Colitis cystica profunda. Am J Gastroenterol 1976;65:339-43.

2. Martin JC, Culp CE, Weiland LH. Colitis cystica profunda. Dis Colon Rectum 1980;23:488-91.

3. Morson BC, Dawson IMP. Gastrointestinal Pathology, 2nd edn. London: Blackwells, 1979:514.

4. Epstein S, Ascari WQ, Ablow RC, Seaman WB, Lattes R. Colitis cystica profunda. Am J Clin Pathol 1966;45:186-201.

5. Herman A, Nabseth D. Colitis cystica profunda. Arch Surg 1973;106:337-41.

6. Silver H, Stolar J. Distinguishing features of well differentiated mucinous adenocarcinoma of the rectum and colitis cystica profunda. Am J Clin Pathol 1969;51:493-500. from other nonintestinal sites can also become localized in the colonic wall, ie, cervix, fallopian tubes and urinary tract (12). Definition of these other entities, however, does not usually present a practical clinical problem in the differential diagnosis of colitis cystica profunda.

Although rare, the diagnosis of colitis cystica profunda should be considered in the evaluation of any inflam-

7. Whitehead R. Mucosal Biopsy of the Gastrointestinal Tract, 4th edn. Philadelphia: WB Saunders Company, 1990:383-4.

8. Wayte DM, Helwig EB. Colitis cystica profunda. Am J Clin Pathol 1967;48:159-69.

9. Magidson JG, Lewin KJ. Diffuse colitis cystic profunda. Report of a case. Am J Surg Pathol 1981;5:393-9.

10. O'Donnell N. Enteritis cystica profunda - revisited. Human Pathol 1987;18:1300-1.

11. Gardiner GW, McAuliffe N, Murray D. Colitis cystica profunda occurring in a radiation-induced colonic stricture. Human Pathol 1984;15:295-8.

12. Levine DS. "Solitary" rectal ulcer syndrome. Are "solitary" rectal ulcer syndrome and "localized" colitis cystica profunda analogous syndromes caused matory process involving the distal colon. It should be carefully considered in any patient with a colonic polypoid mass, especially if multiple endoscopic pinch mucosal biopsies fail to yield a specific diagnosis. Finally, distinction from a mucin-producing colonic adenocarcinoma is particularly important especially if unnecessary and more radical surgery is to be avoided.

by rectal prolapse? Gastroenterology 1987;92:243-53.

13. Bentley E, Chandrasoma P, Cohen H, Radin R, Ray M. Colitis cystica profunda: Presenting with complete intestinal obstruction and recurrence. Gastroenterology 1985;89:1157-61.

14. Fechner RE. Polyp of the colon possessing features of colitis cystica profunda. Dis Colon Rectum 1967;10:359-64.

15. Barner JL. Colitis cystica profunda. Radiology 1967;89:435-7.

16. Carstens PHG, Gonzalez R. Colitis cystica profunda. Acta Pathol Microbiol Scand 1969;75:273-81.

17. Goodall HB, Sinclair ISR. Colitis cystica profunda. J Pathol Bacteriol 1957;73:33-41.

18. Madigan MR, Morson BC. Solitary ulcer of the rectum. Gut 1969;10:871-81. 


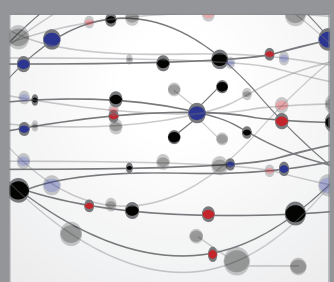

The Scientific World Journal
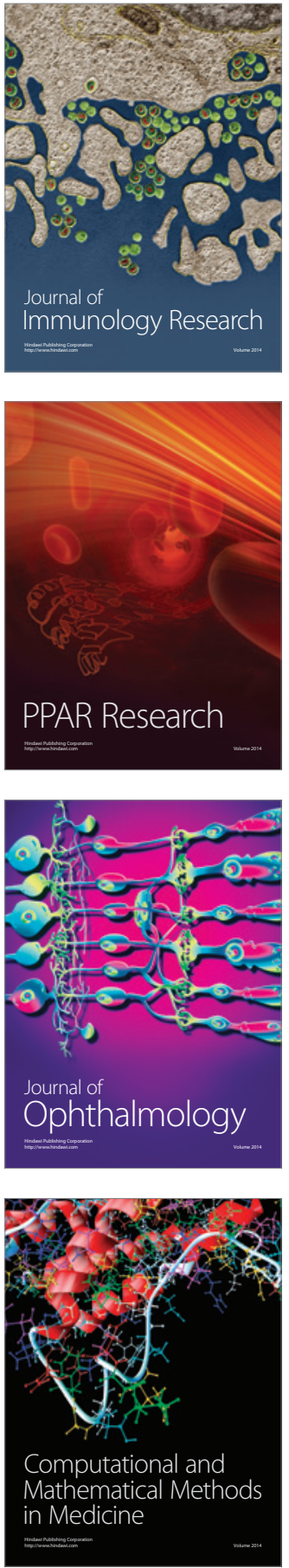

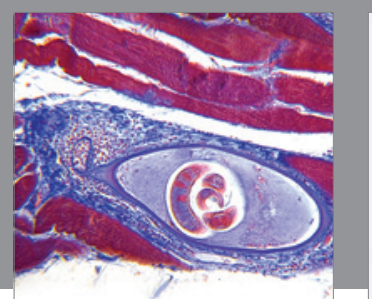

Gastroenterology Research and Practice

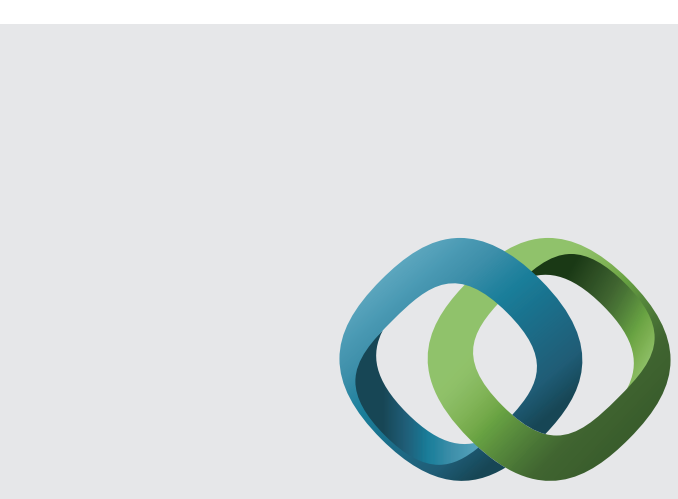

\section{Hindawi}

Submit your manuscripts at

http://www.hindawi.com
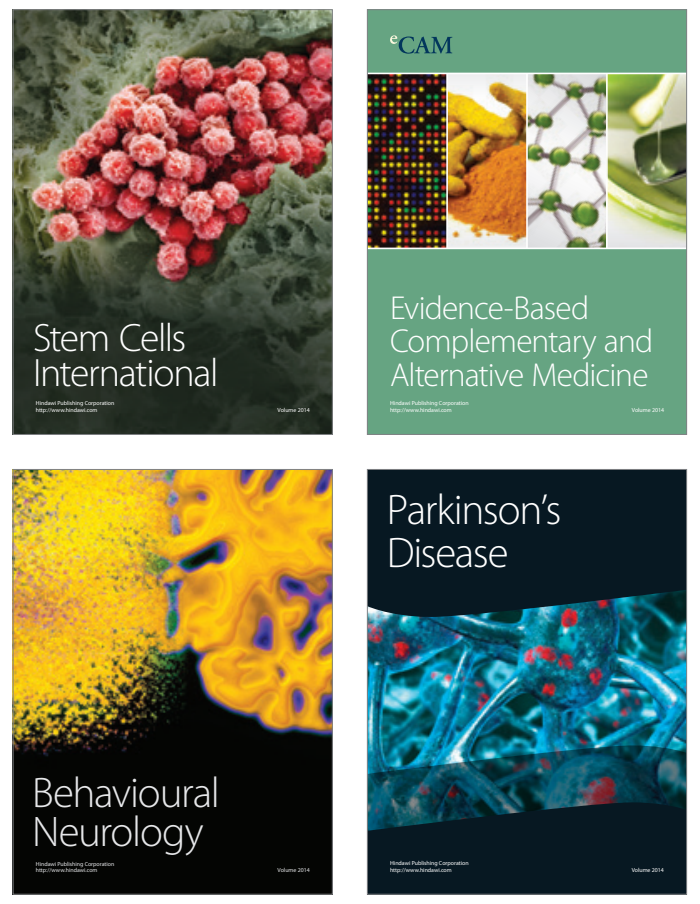
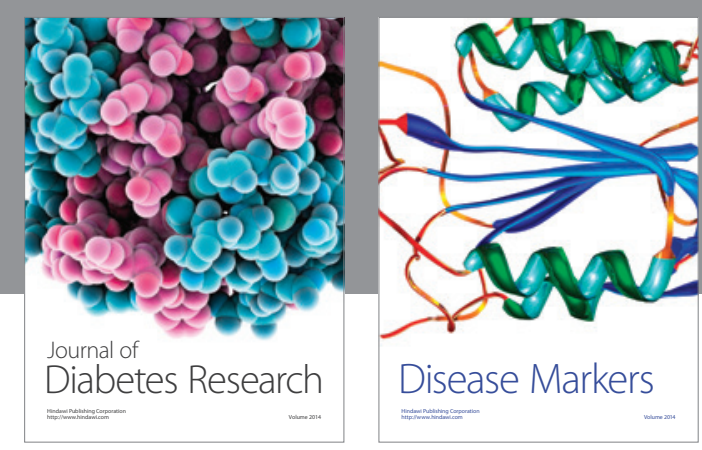

Disease Markers
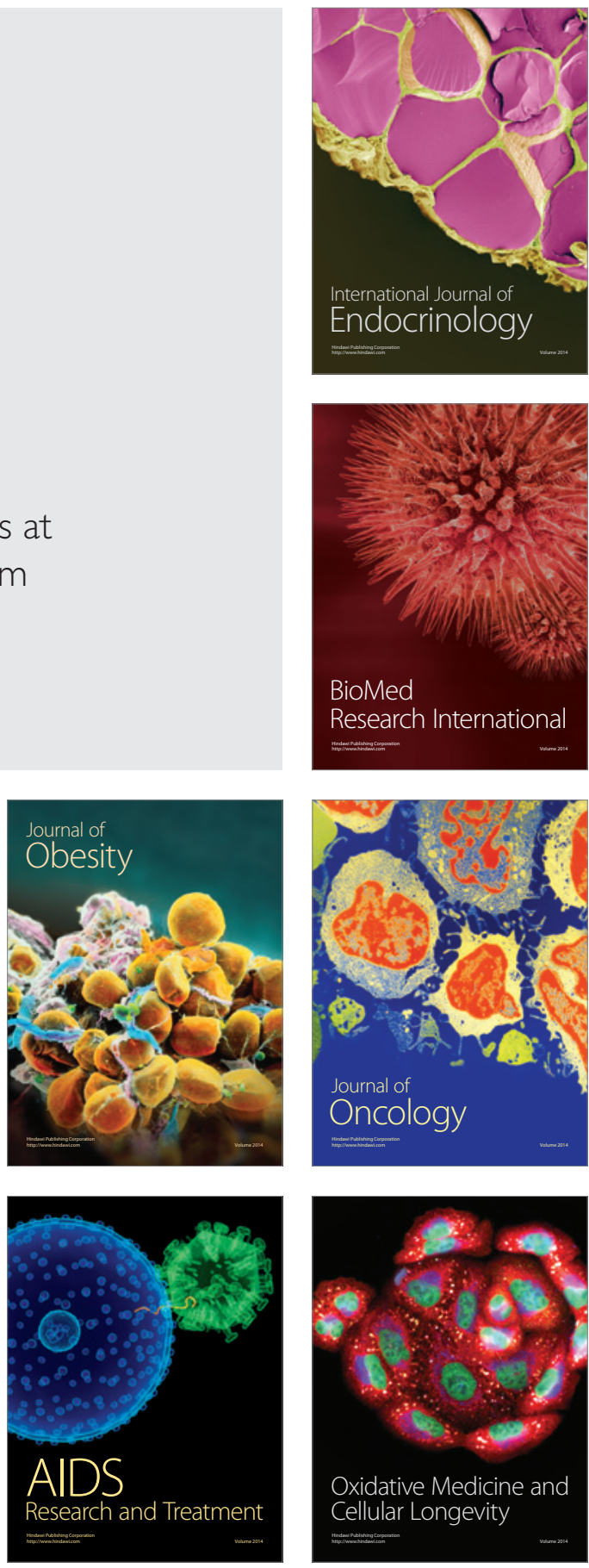